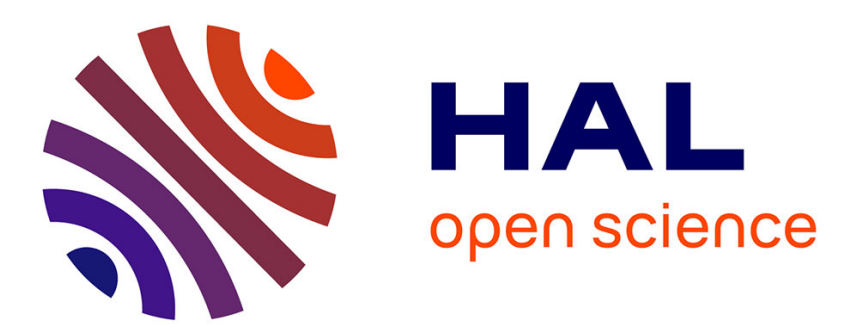

\title{
High BrdU Sensitivity of Passeriformes Chromosomes: Conservation of BrdU-Sensitive Fragile Sites on Their Z Chromosomes during Evolution
}

\author{
Michèle Gerbault-Seureau, Jérôme Fuchs, Bernard Dutrillaux
}

\section{To cite this version:}

Michèle Gerbault-Seureau, Jérôme Fuchs, Bernard Dutrillaux. High BrdU Sensitivity of Passeriformes Chromosomes: Conservation of BrdU-Sensitive Fragile Sites on Their Z Chromosomes during Evolution. Cytogenetic and Genome Research, 2019, 10.1159/000499590 . mnhn-02365422

\section{HAL Id: mnhn-02365422}

\section{https://hal-mnhn.archives-ouvertes.fr/mnhn-02365422}

Submitted on 15 Nov 2019

HAL is a multi-disciplinary open access archive for the deposit and dissemination of scientific research documents, whether they are published or not. The documents may come from teaching and research institutions in France or abroad, or from public or private research centers.
L'archive ouverte pluridisciplinaire HAL, est destinée au dépôt et à la diffusion de documents scientifiques de niveau recherche, publiés ou non, émanant des établissements d'enseignement et de recherche français ou étrangers, des laboratoires publics ou privés. 


\section{High BrdU-sensitivity of Passeriformes chromosomes: conservation of BrdU- sensitive fragile sites on their $\mathrm{Z}$ chromosomes during evolution}

\section{Michèle Gerbault-Seureau, Jérôme Fuchs, Bernard Dutrillaux (a)}

Systématique, Évolution, Biodiversité, ISYEB - UMR 7205 - CNRS MNHN UPMC EPHE, Muséum National d'Histoire Naturelle, Sorbonne Universités, 57 rue Cuvier CP50 F-75005, Paris, France.

(a) Corresponding author bdutrill@mnhn.fr

Key words Turdus - Erithacus - Parus - Z chromosome - BrdU-sensitive - Fragile sites

\section{Summary}

Amongst 15 species of birds, belonging to 7 orders, recurrent breakages evocating the presence of fragile sites were detected in the chromosomes of the 5 species belonging to Passeriformes. These breaks appear when BrdU (5-bromodeoxyuridine) is added to the cell culture medium, at a dose inefficient for inducing chromosome structure alterations in other birds and mammals. They involve, similarly in male and female, 3 loci on the $\mathrm{Z}$ chromosome of 3 species of the genus Turdus (Turdidae). The labeling by BrdU antibody confirms the correlation between BrdU incorporation into DNA and breakage, especially around and in the sites of breakage. Thus, 3 BrdU-sensitive fragile sites are present on the $\mathrm{Z}$ chromosome of these birds. Three fragile sites were also detected at different locations in the $\mathrm{Z}$ chromosomes of the European Robin (Erithacus rubecula Muscicapidae), which suggests that a structural rearrangement occurred during the evolution of Turdidae and Muscicapidae. Chromosome banding confirms this interpretation. Finally, in the more distantly related species Parus major (Paridae), the almost acrocentric $\mathrm{Z}$ chromosome displays a single BrdU-sensitive fragile site on its short arm and the $\mathrm{W}$ appears to be pulverized by BrdU incorporation. Although it cannot be excluded that the BrdU-sensitive fragile sites may be involved in rearrangements, their conservation in many species and possibly all Passeriformes, provides evidence that they do not constitute a pejorative character during evolution.

\section{Introduction}

Fragile sites are specific loci where constitutional chromosome or chromatid gaps, breaks and recombination recurrently occur (ISCN, 1985). Initially described in man, 
they were found later in some other mammals (Bernardino et al. 1983, Gripenberg et al. 1991, Lin et al. 1984, Stone \& Stephens 1993), and recently in birds (Pentzold et al., 2018). Two main classes of fragile sites are acknowledged in the genome: the common fragile sites (CFSs) and the early replicating fragile sites (ERFSs). CFSs are principally induced by replication stress, as that induced by aphidicholine, which allows some portions of the genome to enter mitosis in an under-replicated state (Debatisse et al., 2012). BrdU (5-bromodeoxyuridine)-sensitive fragile sites constitute a category apart and are composed of large A-T rich DNA sequences. Although A-T richness was found in other fragile sites (Zhang et al., 2007), only FRA 10B and FRA12C, located in bands 10q25.2 and 12q24, respectively, are considered as BrdU-sensitive in man (Sutherland et al. 1980). In individuals who do not express the fragility, the sequencing of band $10 \mathrm{q} 25$ revealed the presence of various A-T rich repeats which could reach $52 \mathrm{bp}$ in length (Hewett et al. 1998). The polymorphism, which exists at this site is primary the consequence of the variation of the repeat copy number, which was found elevated in the rare individuals who express the BrdU-sensitivity, compared to the non-expressing population. The BrdU-induced fragility of this AT-rich region is thought to be the consequence of a massive incorporation of $\mathrm{BrdU}$, in place of thymidine. At difference with the carriers of the fragile $\mathrm{X}$ syndrome, no clinical abnormality was noticed in carriers of BrdU-fragile sites, which appear as benign chromosomal markers. Considering their rarity in human, compared to other CFSs, BrdU-sensitive sites are relatively more frequently described than expected in non-human mammals. This may be due to the popularity of BrdU incorporation techniques to induce chromosome banding. They were reported in several Primates, cattle, reindeer, Chinese hamster, dog and mouse (Smeets et al. 1990, Ruiz-Herrera et al. 2002, Bernardino et al. 1983, Gripenberg et al. 1991, Lin et al. 1984, Stone \& Stephens 1993, Helmrich et al. 2006). There is an abundant literature on the chromosomes of birds (Belterman and De Boer 1984, Nie et al. 2015, de Oliveira et al. 2013), including after BrdU treatments, but no fragile sites were noticed (Schmid et al. 1989, Nishida-Umehara et al. 1999). In the course of control chromosome studies of our growing cell bank, we were surprised to detect, in fibroblast cultures treated by low doses of BrdU, multiple recurrent breakages in 2 chromosomes of a male Blackbird Turdus merula Linnaeus, 1758 (Passeriformes: Turdidae). This finding was extended to other birds, and found in other species of 
Passeriformes only. Chromosome banding indicated that these BrdU-inducible fragile sites were located on the sex chromosomes.

\section{Material and methods}

\section{Animals.}

Intercostal muscles were sampled in 14 species in the Centre d'accueil de la Faune Sauvage (CEDAF) rehabilitation center (Ecole Vétérinaire d'Alfort) 4-20 hours after the natural death (Table 1). They included 5 species of Passeriformes, composed of 3 Turdus (Turdidae : Muscicapoidea), one Erithacus (Muscicapidae, Muscicapoidea), one Parus (Paridae, Sylvoidea). Nine other species belonging to 7 different orders were analysed (Table 1). Fibroblasts of a female Gorilla gorilla, which were cultivated by chance at the time of this study, were also used as mammalian control in co-cultures with T. iliacus cells.

\section{Methods.}

Cell Cultures, BrdU treatments, metaphase preparations and chromosome banding were performed according to our usual conditions (Richard et al. 2016) except that BrdU treatments were extended from 4 to $18 \mathrm{~h}$ before harvesting. For co-cultures of G. gorilla (MNHN-TCCV-946) and T. illiacus fibroblasts, the same number of cells from each species was grown together for $6 \mathrm{~h}$ before the synchronization by FdU. For each karyotyped specimen, muscular tissue and cultivated fibroblasts were cryo-conserved in liquid nitrogen. The material was stored in the RBcell (Ressources Biologiques de cellules vivantes: Tissus et Cellules Cryoconservés de Vertébrés (TCCV) collection, at the Muséum National d'Histoire Naturelle of Paris (Table 1).

Evaluation of the AT/GC content of the Z chromosome

BrdU-induced fragility appears to be linked to the number of A-T rich repeats (Voiculescu et al., 1991). To examine if BrdU sensitivity potentially occurs in passerines, we computed the GC content along the $\mathrm{Z}$ chromosome of the Collared Flycatcher (Ficedula albicollis, Muscicapidae; Genbank Acession number CM002017.1) (Ellegren et al. 2012), the only Muscicapoidea for which a reference genome is available, as well as other Passeriformes for which chromosome level assemblies are available (Parus major NC_031799, Laine et al. 2016, Passer domesticus (House Sparrow) CM004527, Elgvin et al, unpublished, and Taeniopyga gutttata (Zebra Finch) NC_011493, Warren et al. 2010). 
GC content plots along the $\mathrm{Z}$ chromosomes were computed using R.3.0.1 (R Core Team 2013) (window size $=1000 \mathrm{bp}$ ).

\section{Results}

Karyotype description and comparison. All the species indicated in table 1 were karyotyped after culture in presence of BrdU during the last S-phase and treatment by the chromosome banding technique described above. Only the results obtained for the following 5 species of Passeriformes, which expressed chromosome fragility, will be described.

Turdus merula (TME, Eurasian Blackbird): Published data: Hammar (1970). The karyotype is composed of 80 chromosomes: 80 , ZZ and 80, ZW in male and female, respectively (Fig 1A). Only the 6 larger autosomes (TME 1 to 6) may be considered as macro-chromosomes. All the others are acrocentric of progressively decreasing sizes. The NORs are located on the telomere region of the long arm of two pairs of microchromosomes. Chromosome $\mathrm{Z}$ is a large sub-metacentric and chromosome $\mathrm{W}$ is a medium sized acrocentric. C-banding intensely stains the centromere regions of chromosomes $1-8, \mathrm{Z}$ and $\mathrm{W}$ and very lightly those of the smaller chromosomes. In addition, the long arm of the $\mathrm{W}$ is C-banded (not shown).

Turdus iliacus (TIL, Redwing): Published data: Derjusheva et al. (2004). The male karyotype is also composed of 80 chromosomes: 80, ZZ (Fig. 1B). Compared to T. merula, chromosomes TIL 1, 2, 4, 6, 7 and 8 look similar to TME 1, 2, 4, 5, 7 and 8, respectively. TIL3 differs from TME 3 and TIL 5 differs from TME 5 by a pericentric inversion. Possible rearrangements involving some of the micro-chromosomes could not be investigated. As in T. merula, the NORs are on 2 micro-chromosomes at the terminal portion of their long arm,

Turdus philomelos (TPH, Song Trush): Published data: none. The male karyotype is composed of 78 chromosomes: 78, ZZ (Fig. 1C). Amongst the 38 pairs of autosomes, 7 are sub-metacentric. Compared to T. iliacus, TPH 1, 2, 5 and 8 look similar to TIL 1, 2, 5 and 8 respectively. TPH 3, more metacentric differs by at least one pericentric inversion from either TIL 3 or TME 3. Pair TPH 4 differs from TIL 4 by the Robertsonian translocation with a micro-chromosome, which possibly was homologous to one of the 2 NOR carrier pairs present in T. merula and T. iliacus. TPH 6 differs from TME 6 and TIL 6 by a pericentric inversion and TPH 7 differs from TME 7 and TIL 7 by an inversion and 
heterochromatin addition. One micro-chromosome and the sub-metacentric chromosomes TPH 4 and 7 are silver stained, all in telomeric position.

Erithacus rubecula (ERU, European Robin): Published data: none. The female karyotype is composed of 80 chromosomes: 80, ZW (Fig. 1D). The number of submetacentric pairs of autosome increases to 11, but the short arms of pairs 7-11 are composed of late replicating, slightly C-banded and polymorphic heterochromatin. After their exclusion, the karyotype of E. rubecula appears to be composed of the same euchromatic content than the species of the genus Turdus. Chromosomes ERU 1, 2, 4 and TME 1, 2, 4 look identical and chromosome ERU 3, 5, 6 and TME 3, 5, 6 differ by one pericentric inversion at least. Both TME Z and ERU Z are sub-metacentric, but they obviously differ by a pericentric inversion: the large R-band distally located on the long arm in TME Z is on the short arm in ERU Z. TME W (acrocentric) and ERU W (submetacentric), both largely composed of C-band positive heterochromatin, also differ by at least one pericentric inversion.

Parus major (PMA, Great Tit): Published data: Hammar (1970). The female karyotype is composed of 80-82 chromosomes: 80/82,ZW (Fig. 2A). The macro-chromosomes 1-6 seem to be homologous to those of the preceding species, but intra-chromosomal changes have occurred. Chromosome 1 is metacentric, as in E. rubecula, chromosome 3 is acrocentric, as in T. merula and chromosomes 4-6 are almost acrocentric, with variable amounts of heterochromatin on their short arm. The short arm of chromosome $\mathrm{Z}$ is very small and recurrently displays one break after BrdU incorporation (Fig. 2A, B, C). The morphology of the $\mathrm{W}$ chromosome is atypical: it looks like a dicentric ring (Fig. 2D). After BrdU treatment, it is intensely labeled by anti-BrdU antibody and displays several breaks (Fig. 2A, B, C).

Demonstration of the BrdU sensitivity. All the following experiments were performed on T. iliacus fibroblasts. They were duplicated in at least one other species, which will not be mentioned unless it provides particular findings.

a) Giemsa stained chromosomes, without BrdU treatment. All chromosomes were homogeneously stained and compacted in the male. In the female, a single macrochromosome occasionally had a particular appearance, with parallel and tightly associated chromatid. It was identified as the W chromosome (largely heterochromatic). 
b) Giemsa stained metaphases after treatment by BrdU. As a rule, BrdU treatments (4 h to $18 \mathrm{~h}$ ) induced much more chromosome gaps and breaks than in mammalian cells at various chromosomal locations (see below). One (in females) or 2 (in males) submetacentrics, identified as the Zs, were systematically broken at 3 sites in genus Turdus. The same observation was done for E. rubecula but the position of the breakages was different. In P. major, a single recurrent break was found on the short arm of the Z. In addition, its chromosome $\mathrm{W}$ was more or less pulverized

c) Co-cultures of male T. iliacus and female G. gorilla cells

In T. iliacus, both Z chromosomes systematically displayed breaks and gaps (an average of 3 per chromosome) in 32/32 analyzed metaphases, in addition to 22 gaps and 2 breaks on autosomes ( $\mathrm{N}^{\circ} 1,2,3$ and 6 or 7$)$. In G. gorilla, only heterochromatin was recurrently elongated. Rarely, one X (the late replicating one) was also elongated, but never broken, and on the whole, autosomes displayed only 4 gaps and 2 chromatid breaks in the 28 analyzed metaphases.

d) Anti-BrdU (green) labeled chromosomes. After 4h BrdU treatment (during the very late S- and G2-phases), anti-BrdU antibodies confirm the correct incorporation of BrdU into DNA, in particular in the fragile sites. In the female of genera Turdus and Erithacus, chromosome W does not display any breakage, but is deeply green labeled (Fig. 3B). This confirms the late replication of this sex chromosome in birds (Schmid et al., 1989), and indicates that breakages induced by BrdU are not simply related to DNA late replication. In the male, the two $\mathrm{Z}$ chromosomes are similarly green labeled, particularly in the 3 regions recurrently elongated or broken (Fig. 3A,B). In E. rubecula (Fig. 3 C) the late replicating heterochromatic arms of chromosomes 8-11 are homogenously labeled, but not especially fragile. The labeling of its $\mathrm{Z}$ chromosome differs from that of other species, which confirms that a structural rearrangement has occurred.

After 6-7h BrdU treatments (during the second half of the S-phase for most of the observed metaphases), the green labeling of all the chromosomes was increased (Fig. 3D and 4A) and formed a G-banding, as expected. Their elongated regions are green labeled. Interestingly, the breaks, observed after Giemsa or PI staining, are often filled by the green staining of the anti-BrdU antibody.

After 18h BrdU treatments (during the whole last cell cycle and eventually a part of the penultimate S-phase) gaps and breaks on both Zs are less frequent and more discreet than after shorter treatments. In some metaphases, one chromatid of each chromosome 
is green, and elongated and the other is reddish and more or less banded (Fig. 4B). On the $\mathrm{Z}$ chromosomes, the green chromatid appears recurrently broken or elongated at the place of the fragile sites. The "broken" fragments, linked by cohesins to the unaltered reddish chromatid remain in place and the green labeling indicates that BrdU rich DNA remains present in the "break". Thus, the lesion may be either a real DNA/chromatid break or a lack of compaction of the chromatin harboring highly BrdU-incorporated DNA, but the causality relationship between BrdU incorporation into DNA and chromatid lesion is obvious.

GC content of the Z chromosome in Passerines. We analyzed the GC content on the Z chromosomes from the 4 species of Passeriformes with informative data (Fig. 5). The global GC content of the $\mathrm{Z}$ was lower than that of the whole genome: Ficedula albicollis : 41.8\% versus 44.29\%; Parus major : 39,3\% versus 41.52\%; Passer domesticus : 40,1\% versus 41.18\%; Taeniopygia guttata: $39.1 \%$ versus $41.82 \%$. In addition, several sites with a null or almost null GC content were found: 5 in Passer domesticus; 3 in Ficedula albicollis and 1 in Parus major. We could study the $\mathrm{Z}$ chromosome of P. major only: its BrdU-sensitive site is in sub-terminal position, as its GC null site is, but we could not know if they are on the same arm. On the whole, sites with a very low GC content occur in the $\mathrm{Z}$ chromosome of Passeriformes, which may represent potential BrdU-sensitive fragile sites.

\section{Discussion}

To the best of our knowledge, the high sensitivity of Passeriformes chromosomes to BrdU, and in particular the highly recurrent breakage of the $\mathrm{Z}$ chromosome, had never been reported. Using a chicken painting probe, Nanda et al. (2008) demonstrated the synteny conservation of the $\mathrm{Z}$ chromosome in birds belonging to 11 families, including Turdidae. Kreschmer et al. (2014) showed that multiple intra-chromosomal rearrangements occurred between two Neotropical species of Turdidae. The 3 species of the genus Turdus studied here, E. rubecula, (Muscicapidae) and P. major displayed alterations of the $\mathrm{Z}$ chromosomes after BrdU incorporation into DNA. Although we show that inversions of the $\mathrm{Z}$ chromosome occurred between Turdus, Erithacus and Parus, the strong conservation of this chromosome in birds (Nanda et al. 2008) suggests that the Z chromosome of many other species of Passeriformes also harbor BrdU-induced fragile sites. The lack of published data on BrdU-induced chromosome fragility prevents to do any generalization about BrdU sensitivity in birds from 
other orders, but our own negative results in 6 orders suggest that BrdU-sensitivity may be limited to Passeriformes.

The demonstration that a BrdU-induced alteration of chromosome structure is a fragile site is not straightforward. Here, we used a low dose of BrdU, but in condition to release the block induced by FudR (5-fluorodeoxyuridine), which probably increased BrdU incorporation into DNA. Indeed, we verified that these low doses have no effect on chromosomes of mammalian cells. High doses of BrdU are much less efficient for altering chromosome compaction when the treatment is prolonged during the whole S-phase, but they slow down the S-phase of the cell cycle. Thus, neither the global incorporation of BrdU into DNA nor the late replication alone is sufficient to induce a breakage or a marked compaction defect, but a massive BrdU incorporation limited to a region with late replicating DNA is. A likely interpretation is that the late replicating fragment of DNA highly enriched in BrdU reaches the G2/M-phase before having completed its replication and looks elongated or broken at metaphase. A similar process may explain the expression of the common fragile sites under replication stress induced by inhibitors such as aphidicolin (Debatisse et al., 2012). By analogy with the FRA10q25 of man, it is postulated that the fragility of the $\mathrm{Z}$ chromosomes of the birds studied here is due to the presence of large AT-rich repeats, distributed at 3 locations in genera Turdus and Erithacus and a single one in P. major. This interpretation is compatible with the data on the DNA GC content of some species of Passeriformes, which shows the existence of GC null fragments.

\section{Acknowledgments}

We are very grateful to Cécile Le Barzic, Pascal Arné, Jean-Francois Courreau, Miyuki Monty and Annabelle Roy (CEDAF) for their essential collaboration and help for this project. G. gorilla fibroblasts were obtained from the Zooparc of Beauval (France).

\section{Statements of ethics}

All birds that were used in this study either died from their injuries when entering the rehabilitation, or were euthanized, by authorized staff of the CEDAF (Ecole Vétérinaire de Maisons-Alfort, France), because they could not be released in the wild.

\section{Disclosure Statement}

All authors declare that they have no conflicts of interest 


\section{References}

Belterman RHR, De Boer LEM: A karyological study of 55 species of birds, including karyotypes of 39 species new to cytology. Genetica 65: 39-82 (1984).

Bernadino di D, Iannuzi G, Meo di GP: Localization of BrdU-induced break sites in bovine chromosomes. Caryologia 36: 285-292 (1983).

Debatisse M, Le Tallec B, Letessier A, Dutrillaux B, Brison 0: Common fragile sites: mechanisms of instability revisited. Trends in Genetics 28: 22-32 (2012).

de Oliveira EHC, Tagliarini MM, dos Santos MS, O’Brien PCM, Ferguson-Smith MA: Chromosome painting in three species of Buteoninae: A cytogenetic signature reinforces the monophyly of South American species. PLoS ONE 8 (7) e70071 (2013).

Derjusheva S, Kurganova A, Habermann S, Gaginskaya E: High chromosome conservation detected by comparative chromosome painting in chicken, pigeon and passerine birds. Chromosome Res 12: 15-723 (2004).

Ellegren H, Smeds L, Burri R, Olason Pl, Backström N, Kawakami T, Künstner A, Mäkinen H, Nadachowska-Brzyska K, Qvarnström A, Uebbing S, Wolf JB: The genomic landscape of species divergence in Ficedula flycatchers. Nature 491: 756-760 (2012).

Gripenberg U, Huuhtanen S, Wessman M, Nieminen M: A fragile site in the X chromosome of the reindeer (Rangifer tarandus L) Genet Sel Evol 23 suppl1, 135s-139s (1991).

Hammar B: The karyotype of thirty-one birds. Hereditas 65: 29-58 (1970).

Helmrich A, Stout-Weider K, Hermann K, Schrock E, Heiden T: Common fragile sites are conserved features of human and mouse chromosomes and relate to large active genes. Genome Res 16: 1222-1230 (2006). 
Hewett DR, Handt O, Hobson L, Mangelsdorf M, Eyre HJ, Baker E, Sutherland GR, Schuffenhauer S, Mao J, Richards RI: FRA10B structure reveals common elements in repeat expansion and chromosomal fragile site genesis. Mol Cell 1: 773-781 (1998).

Kretschmer R, Gunski RJ, Del Valle Garnero A, de Oliveira Furo I, O’Brien PCM, FergusonSmith MA, Correa de Oliveira EH: Molecular cytogenetic characterization of multiple intrachromosomal rearrangements in two representatives of the genus Turdus (Turdidae, Passeriformes). PLoS One 2014; 9 (7)e103338

Laine VN, Gossmann TI, Schachtschneider KM et al. : Evolutionary signals of selection on cognition from the great tit genome and methylome. Nat Commun 7: 10474 (2016) Doi: 10.1038/ncomms 10474

Lin MS, Takabayashi T, Wilson MG, Marchese CA: An in vitro and in vivo study of a BrdUsensitive fragile site in the Chinese hamster. Cytogenet Cell Genet 38: 211-215 (1984).

Nanda I, Schlegelmilch K, Haaf T, Scharti M, Schmid M: Synteny conservation of the Z chromosome in 14 avian species (11families) supports a role for $\mathrm{Z}$ dosage in avian sex determination. Cytogenet Genome Res 122: 150-156 (2008).

Nie W, O’Brien PCM, Wang J, Su W, He K, Bed'Hom B, Volobouev V, Ferguson-Smith MA, Dobigny G, Yang F: Multidirectional chromosome painting substantiates the occurrence of extensive genomic reshuffling within Accipitriformes. BMC Evol Biol 15: 205 (2015) DOI 10.1186/s12862-015-0484-0

Nishida-Umehara C, Fujiwara A, Ogawa A, Mizuno S, Abe S, Yishida MC: Differentiation of $\mathrm{Z}$ and $\mathrm{W}$ chromosomes revealed by replication banding and FISH mapping of sexchromosome-linked DNA markers in the cassowary (Aves, Ratitae). Chromosome Res 7: 635-640 (1999).

Pentzold C, Shah SA, Hansen NR, Le Tallec B, Seguin-Orlando A, Debatisse M,Lisby M, Oestergaard VH; FANCD2 binding identifies conserved fragile sites at large transcribed genes in avian cells. Nucleic Acids Res 46: 1280-1294 (2018). 
R Core Team: R: A language and environment for statistical computing. R Foundation for Statistical Computing, Vienna, Austria. Available via http://www.R-project.org/ (2013).

Richard F, Gerbault-Seureau M, Bounneuang Douangboupha Keovichit K, Hugot JP, Dutrillaux B : Karyotype diversity suggets that Laonastes aenigmamus (Laotian rock rat)(Rodentia, Diatomyidae) is a multi-specific genus. Chromosome Res 24: 379-392 (2016). DOI 10.1007/s10577-016-9527-7

Ruiz-Herrera A, Ponsa M, Garcia F, Egozcue J, Garcia M, Ponsa M, Garcia F, Egozcue J: Fragile sites in human and Macaca fascicularis chromosomes are breakpoints in chromosome evolution. Chromosome Res 10: 33-44 (2002).

Schmid M, Enderle E, Schindler D, Schempp W: Chromosomal banding and DNA replication patterns in bird karyotypes. Cytogenet Genome Res 52: 139-146 (1989).

Smeets DF, van de Klundert FA, van de Klundert FA : Common fragile sites in man and three closely related primate species. Cytogenet Cell Genet 53: 8-14 (1990).

Stone DM, Stephens KE: Bromodeoxyuridine induces chromosomal fragile sites in the canine genome. Amer J Med Genet 15: 198-202 (1993).

Sutherland GR, Baker E, Seshadri RS: Heritable fragile site on human chromosomes. A new class of fragile site requiring BrdU for expression. Am J Hum Genet 32: 542-548 (1980).

Voiculescu I, Back E, Schempp W: Homozygous condition for a BrdU-requiring fragile site on chromosome 12. Hum Genet 86: 416-417 (1991).

Warren WC, Clayton DF, Ellegren H: The genome of a songbird. Nature 464: 757-762 (2010). 
Zhang H, Freudenreich CH: An AT-Rich sequence in human common fragile site FRA16D causes fork stalling and chromosome breakage in S. cerevisiae. Molecular Cell 27: 367379 (2007).

\section{Legends}

Table 1 List of birds, with reference numbers, in which we observed (BrdU-s +) or did not observe (BrdU-s -) fragile sites on the sex chromosomes after BrdU treatment during the last S-phase.

Figure 1 RHG-banded karyotypes after BrdU-treatment and FPG staining (breaks on the Zs are indicated by arrow. A) female karyotype of Turdus merula. B) male karyotype of Turdus iliacus. Silver-stained chromosomes 21 and 22 from the same cell are added: NORs are indicated by arrow-heads. C) male karyotype and silver-stained chromosomes 4, 7 and 24 of Turdus philomelos. NORs are indicated by arrow-heads. D) female karyotype of Erithacus rubecula. Heterochromatic short arms of chromosomes 7-11 vary in size and are late replicating.

Figure 2 A) RHG-banded female karyotype after BrdU treatment of Parus major. B, C, D) $\mathrm{Z}$ and $\mathrm{W}$ chromosomes from other cells. Breaks are indicated by arrows. D) Sequentially Giemsa stained and C-banded sex chromosomes (no BrdU-treatment) showing the unusual morphology of the W.

Figure 3 Metaphases obtained after $4 \mathrm{~h}(\mathrm{~A}, \mathrm{~B}, \mathrm{C})$ and $6 \mathrm{~h}(\mathrm{D})$ treatments by BrdU and stained by PI (red) and antibody anti-BrdU (green). A) Male Turdus iliacus, B) Female Turdus merula. C) Female Erithacus rubecula. D) Female Parus major. Breaks on the Z chromosome are masked by the green fluorescence (see next figure).

Figure 4 Turdus iliacus A) Male metaphase obtained after a 6h BrdU treatment stained by propidium iodide (PI) and anti-BrdU antibody. Only PI stained Zs (white) display the BrdU-induced breaks (arrows). B) Male metaphase of Turdus iliacus obtained after 18h BrdU treatment (2 S-phases), and stained by PI (red) and anti-BrdU antibody (green). The green chromatids are elongated (incorporation during $2 \mathrm{~S}$-phases) and their BrdU- 
induced "breaks" on the Zs are visible only after suppression of the green staining (shown by PI staining on white chromosomes).

Figure 5 GC content plots along the Z chromosomes of Ficedula albicolis, Parus major, Taeniopygia guttata and Passer domesticus. Plots were computed using R.3.0.1 (R Core Team, 2013) and a window size $=1000$ bp. Nucleotide positions are in bp and refer to the position on the assembled Z chromosome (www.ncbi.nlm.nih.gov). Sites with a null or very low G-C content represent potential BrdU-sensitive fragile sites. 